\title{
More Questions Than Answers: Factors Affecting Human Gender Identity and Sexual Orientation: A Mini Review
}

\author{
Des Gilmore* \\ Laboratory of Human Anatomy, University of Glasgow, Scotland \\ *Corresponding author: Des Gilmore, Laboratory of Human Anatomy, College of Medical, Veterinary \& Life Sciences, University of \\ Glasgow, Glasgow G12 8QQ, Scotland. \\ To Cite This Article: Des Gilmore, More Questions Than Answers: Factors Affecting Human Gender Identity and Sexual Orientation: A Mini Review. \\ 2020 - 10(2). AJBSR.MS.ID.001542. DOI: 10.34297/AJBSR.2020.10.001542.
}

Received: 制 October 07, 2020; Published: 眥 October 16, 2020

\begin{abstract}
One's gender identity and sexual orientation are determined by a combination of factors. These include a genetic predisposition, hormonal activity during fetal and neonatal life and sensitive periods during childhood when the capacity for establishing sexual preferences are subject to influences from both family and society. Animal studies have shed some light on the situation, as also have anomalies that have arisen during human development. However, there is still much to be learnt about how this takes place.
\end{abstract}

\section{Introduction}

Currently there has probably never been more interest in brain development and how one's genetic makeup, hormones and the environment, acting during limited critical periods, affect one's future gender identity, thoughts, emotions and consequent behavior. However, the topic has now become highly politicized and it is thus a difficult time to express opinions, even if these are based on robust scientific research findings, without offending one group or another. As a scientist working in the field of reproductive endocrinology over many years, I strongly believe that it is logical to recognize that sexual dimorphism does indeed exist in the central nervous system (CNS) despite vehement denials by others with strongly held views on the subject $[1,2]$. Sexual dimorphism is evident in many structures in the body including muscle development, height and skeletal anatomy. In fact, every cell in the mammalian body differs between the sexes, being either XX or XY; the haploid germ cells being either $\mathrm{X}$ or $\mathrm{Y}$ in the male and just $\mathrm{X}$ in the female. However, in no way whatsoever does this make one gender superior of inferior to the other.

There is a plethora of well-documented findings confirming sexual dimorphism in the CNS and how in animals, manipulation of the hormonal environment during the aforementioned critical periods in development can have far-reaching and long-lasting consequences (for recent reviews see [3,4]). However, the human brain is far more complex than that of animals and thus much of what we know about the situation here has been largely learnt by observing the effects of anomalies that occur during development.

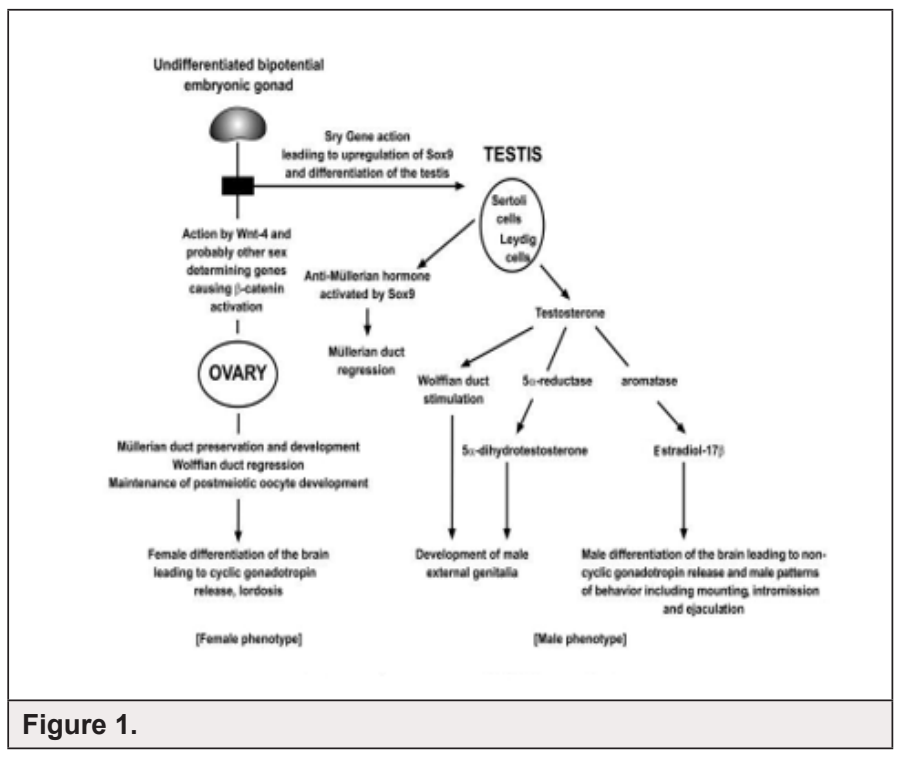

In the human fetus the testes are active from the eighth week of gestation, stimulated by the action of human chorionic 
gonadotropin (hCG) from the placenta. Male differentiation of the external genitalia is brought about at this time largely by the conversion of testosterone, produced in the Leydig cells, to its more active metabolite $5 \alpha$-dihydrotestosterone ( $5 \alpha$-DHT) (Figure 1). Later during fetal life and in the newborn, masculinization of the CNS takes place through the action of testosterone itself. This leads to a marked gender difference in the size of some groups of nerve cells (nuclei) in the brain, although not all are associated with reproductive behavior. It is thus obvious that sexual differentiation of the CNS, although more subtle than that of the genitalia, is just as important. Problems may arise when there is a discrepancy between sexual differentiation of the genitalia and that of the brain.

\section{Brain Sexual Differentiation}

Numerous studies upon rodents have demonstrated that in those regions of the CNS where steroid hormone receptors are present, testosterone crosses the blood brain barrier and as a result of intraneuronal aromatization to estradiol-17 $\beta$ (E2) masculinization of the brain takes place. It appears in rodents the female brain is largely prevented from masculinization by the circulating E2 being strongly bound to $\alpha$-fetoprotein and therefore unable to enter into the neurons. In primates it has been postulated that it is sex hormone binding globulin (SHBG) that binds E2, thus preventing it from passing into the neurons of the developing female brain [5]. However, an alternative view regarding brain masculinization in humans has recently been put forward [6]. This is that a functional androgen and not an estrogen receptor in the CNS is required for the developing brain to be programmed for future male sexual orientation and behavior. Moreover, it has been pointed out that men lacking the aromatase enzyme still show masculine behavior despite their inability to aromatize testosterone to E2 [7].

Sex differences during development in exposure to glucocorticoids are also important as has been pointed out [8]. Near the end of pregnancy in humans, both sexes experience a critically timed rise in the bioavailability of glucocorticoids released from the fetal/maternal adrenal axis. It has been emphasized that in males, but not females, there may be competing epigenetic influences, as the glucocorticoids briefly overlap with testosterone or estrogenic metabolites on organizational processes in the CNS, thus contributing to its sexual dimorphism. If, for some reason, the glucocorticoids are inappropriately elevated either by the mother being severely stressed, or by the administration of several doses of exogenous glucocorticoids when premature birth is threatened, brain structure and function may be affected leading to a varying degree of feminization of reproductive behavior in later life. Moreover, the hypothalamic-pituitary-adrenal axis reactivity to stress can also be impaired and consequently physiological stress-coping mechanisms. It is thus evident that both the sex and stress hormones play a vital role in brain development, regulating the number, site and connectivity of neurons by influencing neurogenesis, apoptosis, survival, migration and synaptogenesis. An impairment of stress-coping mechanisms during development, associated with a faulty programming of the hypothalamic-pituitary adrenal axis, is a risk factor for the appearance of psychiatric and other brain disorders in later life, some of which show clear sex differences.

In animals with short gestation periods, such as many rodents, it is in the perinatal period that androgens cause the sexual dimorphism seen in the brain and spinal cord. Only during this time is neural tissue sufficiently plastic to respond irreversibly to the actions of these hormones. Such critical periods are merely empirical concepts and do not represent clearly defined stages in development. It also cannot be assumed that all sexually differentiated CNS structures are maximally sensitive to steroids within the same time period and may differ temporally for various sexually dimorphic traits. Manipulation of the hormonal environment in rodents and other animals during these periods can bring about permanent alterations in brain structures and later in reproductive behavior. One can thus effectively convert a male brain into a female one and vice versa.

\section{Differentiation of Sexual Behavior}

Neurotransmitters as well as the endogenous opioids, play a vital role in regulating many functions in the brain. Some decades ago we demonstrated that that in rats the administration of androgens during early life had long-lasting effects in several brain regions, including those not involved in the regulation of reproductive function $[9,10]$. The treatment led to sex differences in the content of certain neurotransmitters including norepinephrine, epinephrine, dopamine and serotonin (5-HT). Shortly after, we were able to show that, when central 5-HT levels are reduced in male hamsters around the time of sexual differentiation of the CNS, female sexual behavior is increased in these animals when adults [11]. Exposure to opiates has been shown to interfere with neural development [12]. When we administered the long-acting opiate (Duromorph®) to golden hamsters during the last four days of their pregnancy and to their offspring within the first few days following their birth, adult sexual behavior in the males, although not the females was drastically altered. In the former both masculine and unexpectedly also feminine sexual behavior were very significantly increased [13,14]. Extrapolation of these findings to the human situation could have implications for the children born to women taking heroin or other opiates during pregnancy.

As in other animals, many sexually dimorphic areas exist in the primate brain and spinal cord; these are not always associated with reproductive behavior. In humans differences between males and females have been recorded in such regions as the anterior hypothalamus the supraoptic and suprachiasmatic nuclei, 
the hippocampus, vomeronasal organ, bed nucleus of the stria terminalis, the anterior commissure and the corpus callosum (see 4 for more detail). This is unsurprising and being aware of such differences is of vital importance in the treatment of many medical conditions. These include neurodegenerative diseases including Parkinson's and Huntington's as well as the consequences of brain injury including stroke.

It has now been clearly demonstrated that sexually dimorphic behavior is recognizable even in infants, for example in play situations, and that this has been imprinted on the brain prior to birth [15]. Studies on rhesus and vervet monkeys have indicated this also takes place in non-human primates $[16,17]$. Such gender different behaviour is apparent too in cognitive and emotional responses to learning, memory, language, fear, anxiety and proprioception [18]. Many of the actions of androgens upon the brain early in development will, however, not become evident till around puberty. What implications do these have for one's quality of life and place in society? In the former case it is important to be aware that gender differences are associated with certain pathologies [19]. Stuttering, dyslexia, attention deficit hyperactivity disorder and autism are much more likely to be recognized in boys than in girls, although they are certainly underdiagnosed in the latter. Criminality, especially in association with aggression and violence, is much more common in men than in women. Lefthandedness is more frequently seen in men [see 4] and may be associated with immunological problems; there is also a slightly higher incidence of homosexuality amongst both left-handed men and women.

\section{Gender Identity}

In humans there is a rare condition known as androgen insensitivity or testicular feminization syndrome. The androgen receptors in the tissues of these individuals are unresponsive to male hormones, which leads to them being identified as girls at birth. It is often not till puberty is delayed that investigations reveal that the person is genetically male although with female external genitalia and gender identity, as neither have been masculinized during early development. Such women tend to be very beautiful, as typified by the 1930s actress Jean Harlow. Another recent example is the Belgian model Hanne Gabe Odiele. In girls born with congenital adrenal hyperplasia some masculinization may have occurred during fetal life, affecting to a varying degree both the genitalia and brain. An increased incidence of homosexuality is often seen in these women. Further light has been shed on the determinants of one's sexual orientation and gender identity by studying those rare instances when infant boys have been reassigned as girls following damage to the penis. Such children have been found to have major problems accepting their newly assigned gender as their brains have already been masculinized. Observations on groups of related individuals in the Dominican Republic and later in Papua New Guinea have provided more insight into this situation [20,21]. Boys born there with an enzyme deficiency preventing them from converting testosterone to $5 \alpha$-DHT have incompletely developed male genitalia. Nevertheless, their brains have been masculinized by testosterone. Although many of these boys were raised as girls, they had less difficulty than might be expected in adopting a masculine gender reassignment at puberty as their programed gender identity was already male.

Children are generally well aware of their gender identity and sexual orientation long before puberty. One only needs to recall their own childhood thoughts and experiences to realize this. It seems logical to assume that variations in circulating levels of androgens during fetal life can influence one's predisposition to later being predominantly hetero- or homosexual, Nevertheless, environmental influences such as parental views and gender differential treatment are also believed to have some influence on one's gender identity. Recently too evidence has accumulated to indicate that a gene or genes may influence, at least to some extent, one's sexual orientation without necessarily determining it [21]. Moreover, often due to pressures from family and society, individuals realizing they are homosexual, or transgender have been forced, or strongly persuaded, into adopting a heterosexual lifestyle. Unfortunately, even today prosecution and persecution of such individuals are still regarded as acceptable in many societies and countries.

With regards to individuals identifying as transsexual, this may be explained at least partly by a divergence between their anatomical gender assigned at birth and their CNS having developed along a different pathway. Thus, one's own personal experience of gender may not always correlate with the assigned anatomical sex. However, it must be emphasized that no gender identity is ever of less value than another, but in many instances, problems have arisen because society does or cannot understand the situation. In the UK the Equality Act of 2010, relating to England and Wales, states that you must not be discriminated against because you are transsexual, when your gender identity is different from the gender assigned to you when you were born. Under the Act, to be prevented from gender reassignment discrimination, it is not necessary to have undergone any specific treatment or surgery to alter your sex applied at birth to that of your preferred gender. Nevertheless, there are certain circumstances when being discriminated against due to gender reassignment is considered lawful. Examples are those rare instances when accessing a service provided only for men or just for women, or in those relating to some competitive sports. Difficulties have arisen in the latter situation regarding the eligibility of trans sportspeople to complete in binary events. Even in cases where surgical reassignment has taken place trans women may be prevented from participating as female competitors. This 
is because it is believed that in some circumstances other women may be disadvantaged compared to those who transitioned to their true gender post-pubertally. Now, in many instances, a third category has been accepted in athletics for non-binary competitors. However, it must be borne in mind too that non-binary gender identities are independent of one's sexual orientation. The limits to what is considered acceptable by many trans individuals, in an attempt to protect women's rights and women only spaces, have themselves caused a lot of controversy and there have been strong arguments for the Act to be reformed and updated. Opponents to change argue that a balance must be achieved between women's rights and concerns and a fear of abuse by the very small number of men self-identifying as trans individuals in order to gain access to women's sex-segregated areas. However, many trans people feel it is their rights that are not being taken into account. Obviously, no single ruling will satisfy everyone.

\section{Conclusions}

It mustalways be kept in mind that human sexual behavior is very complex with there probably being no limit to the variety of human sexual lifestyles thus confirming that one's sexual orientation is not exclusively fixed, but somewhat flexible. There are sensitive periods during fetal and very early neonatal life, as well as in childhood, when the capacity for establishing sexual preferences is maximal, leading to the development of either a primarily heterosexual, homosexual, bisexual or asexual orientation. Nevertheless, exclusive heterosexuality and exclusive homosexuality lie at the opposite ends of a range of sexual preferences laid down by a complex set of genes inherited from both parents and influenced by the internal and external environment during development. As our knowledge and understanding about gender identity and sexual orientation expands, hopefully also will be an increase in the understanding and acceptability of everyone irrespective of their lifestyle.

\section{References}

1. Rippon G (2019) The Gendered Brain. Bodley Head pp. 448. ISBN13.9781847924766

2. Fine C (2010) Delusions of Gender. WW Norton \& Company pp. 338. ISBN 0393968382

3. Gilmore DP (2010) Human brain sexual dimorphism: causes and consequences. Neurobiologia 73: 19-33.

4. Gilmore DP (2015) Sexual Dimorphism in the Central Nervous System Chapter 1 pp.1-30 in Mammalian Endocrinology and Male Reproductive Biology Edited by Shio Kumar Singh CRC Press pp. 331. ISBN-13:978-1 4987-2735-8.

5. Hong H, Branham WS, Ng HW, Moland CL, Dial SL, Fang H, et al (2015) Human sex hormone binding globulin binding affinities of 125 structurally diverse chemicals and comparison with their binding to androgen receptor, estrogen receptor and alpha-fetoprotein. Toxicol Sci 143(2): 333-348.

6. Motta-Mena NV, Puts D (2018) Endocrinology of human sexuality, mating and reproductive behaviour. Horm Behav 91: 19-35.

7. Puts D, Motta-Mena NV (2018) Is human brain masculinization estrogen receptor-mediated? Reply to Luoto and Rantala. Horm Behav 97: 2-4.

8. Gillies GE, Virdee K, McArthur S, Dalley JW (2014) Sex-dependent diversity in ventral tegmental dopaminergic neurons and developmental programing: A molecular, cellular and behavioral analysis. Neuroscience 282: 69-85.

9. Siddiqui A, Gilmore DP (1988) Regional differences in the catecholamine content of the rat brain: effects of neonatal castration and androgenization. Acta Endocrinol Cop 118(4): 483-494.

10. Siddiqui A, Gilmore DP, Clark J (1989) Regional differences in the indoleamine content of the rat brain: effects of neonatal castration and androgenization. Biogenic Amines 6: 105-114.

11. Johnston HM, Payne AP, Gilmore DP, Wilson CA (1990) Neonatal serotonin manipulation alters the adult sexual behaviour of golden hamsters. Pharmacol Biochem Behav 35: 571-575.

12. Ricalde AA, Hammer RP (1990) Perinatal opiate treatment delays growth of cortical dendrites. Neuroscience Letters 115: 137-143.

13. Johnston HM, Payne AP, Gilmore DP (1992) Perinatal exposure to morphine affects adult sexual behaviour of the male golden hamster. Pharmacol Biochem Behav 42(1): 41-44.

14. Johnston H, Payne AP, Gilmore DP (1994) Effect of exposure to morphine throughout gestation on feminine and masculine adult sexual behaviour in golden hamsters. J Reprod Fert 100(1): 173-176.

15. Bao A-M, Swaab DF (2011) Sexual differentiation of the human brain: Relation to gender identity, sexual orientation and neuropsychiatric disorders. Frontiers in Neuroendocrinology 32(2): 214-226.

16. Aexander GM, Hines M (2002) Sex differences in response to children's toys in nonhuman primates (Cercopithecus aethiops sabaeus). Evol Hum Behav 23: 467-479.

17. Hassett JM, Siebert ER, Wallen K (2008) Sex differences in rhesus monkey toy preferences parallel those of children. Horm Behav 54(3): 359-364.

18. McCarthy MM, Arnold AP, Ball GF, Blaustein JD, De Vries GJ (2012) Sex differences in the brain: The not so inconvenient truth J Neurosci 32(7): 2241-2247.

19. Baron-Cohen S, Lombardo MV, Auyeung B, Ashwin E, Chakrabarti B, Knickmeyer R (2014) Why are autism spectrum conditions more prevalent in males? Proc Natl Acad Sci USA 111: 823-828.

20. Imperato-McGinley J, Peterson RE, Gautier T, Sturla E (1979) Androgens and the evolution of male gender identity among male pseudohermaphrodites with $5 \alpha$-reductase deficiency. New Engl J Med 300(22): 1233-1237.

21. Imperato-McGinley J, Miller M, Wilson JD, Peterson RE, Shackleton C, et al. (1991) A cluster of male pseudohermaphrodites with $5 \alpha$-reductase deficiency in Papua New Guinea. Clin Endocrinol 34(4): 293-298.

22. Sanders AR, Martin ER, Beecham GW, Guo S (2015) Genome wide scan demonstrates signjficant linkage for male sexual orientation. Psychol Med 45(7): 1379-1388. 